\title{
Dopamine transporter changes after unilateral deep brain stimulation in progressive Parkinson's disease: a case report
}

This article was published in the following Dove Press journal:

Neuropsychiatric Disease and Treatment

8 April 2014

Number of times this article has been viewed

\author{
Huiwei Zhangl,* \\ Yuanyuan Wang I,* \\ Jinchuan Liang ${ }^{2}$ \\ Stefan Förster ${ }^{3}$ \\ Ping $\mathrm{Wu}^{\prime}$ \\ Jun Zhao' \\ Yihui Guan' \\ Chuantao Zuo' \\ 'PET Center, Huashan Hospital, \\ Fudan University, ${ }^{2}$ Department of \\ Neurosurgery, Changhai Hospital, \\ Shanghai, People's Republic of \\ China; ${ }^{3}$ Department of Nuclear \\ Medicine and TUM-Neuroimaging \\ Center, Technical University Munich, \\ Munich, Germany \\ *These authors contributed equally to \\ this work
}

Correspondence: Chuantao Zuo PET Center, Huashan Hospital, Fudan University, 12 Wulumuqi Middle Road, Shanghai 200040, People's Republic of China

Tel +86 2l 64285263

$\mathrm{Fax}+862164283265$

Email zuoct_cn2000@I26.com

\begin{abstract}
Deep brain stimulation (DBS) at the subthalamic nucleus has been approved as an effective treatment for refractory symptoms of Parkinson's disease (PD). Studies have shown that bilateral DBS surgery in PD patients results in clinical improvement without reducing dopamine transporter function. Here, we report our longitudinal findings in one PD patient, ie, decreases in striatal dopamine transporter binding during one year of follow-up after unilateral DBS at the subthalamic nucleus. Based on this case, we hypothesize that clinical benefit after unilateral DBS may be not directly associated with changes in function at the subthalamic nucleus.

Keywords: deep brain stimulation, Parkinson's disease, dopamine transporter, positron emission tomography
\end{abstract}

\section{Introduction}

Deep brain stimulation (DBS) has been approved as an effective treatment for refractory symptoms of Parkinson's disease (PD). It has been shown that DBS has clinical benefit for PD patients and may reduce the need for anti-parkinsonian medication. Studies have shown that bilateral DBS surgery in PD patients results in clinical improvement without reducing dopamine depletion. ${ }^{1-3}$ Most of these studies have used ${ }^{123}$ ioflupane single-photon emission computed tomography ( ${ }^{123}$ I- FP-CIT SPECT) with its relatively low spatial resolution to investigate distribution of the dopamine transporter in the subregions of the basal ganglia. It is noteworthy that conclusions regarding the changes in dopamine transporter distribution have not been directly evidence-based, nor relied on direct comparison of decreases in the dopamine transporter between DBS patients, levodopa-responsive PD patients with advanced PD, or natural decline. Until now, no clinical ${ }^{11} \mathrm{C}-2 \beta$-carbomethoxy-3 $\beta$-(4-fluorophenyl) tropane positron emission tomography $\left({ }^{11} \mathrm{C}-\mathrm{CFT} \mathrm{PET}\right)$ study has been reported focusing on comparison of dopamine degeneration in each cerebral hemisphere after unilateral DBS to the subthalamic nucleus. We report the longitudinal findings for one such PD patient, with observation of decreases in the striatal dopamine transporter ratio during one year of follow-up after unilateral DBS to the subthalamic nucleus.

\section{Case report}

A 56-year-old right-handed female PD patient with no family history of PD developed early symptoms of rigidity and mild tremor on the right side in 1995. She accepted left pallidotomy in 1999. After the surgery, pharmacological therapy was started using sustained-release carbidopa-levodopa, adamantanamine, and benzhexol 
hydrochloride to control her symptoms. By February 2011, her medication was proving ineffective, so the surgeons accepted her as a candidate for DBS to the subthalamic nucleus. In 2011, the motor symptoms in her left limbs were more severe than in the right limb. Her Unified Parkinson's Disease Rating Scale (UPDRS) score was 19 in the left limb and 11 in the right. After considering the financial circumstances of her family, the surgeons chose to treat the right subthalamic nucleus.

In February 2011, ${ }^{11} \mathrm{C}$-CFT PET was performed after 12 hours off medication to observe dopamine transporter distribution. On the following day, the patient underwent unilateral DBS surgery to the right subthalamic nucleus. In January 2012, a follow-up ${ }^{11}$ C-CFT PET scan was performed in the "electrodes on" state after 12 hours off medication to assess the longitudinal effect of DBS on the subthalamic nucleus. The patient's UPDRS and Hoehn and Yahr scores were assessed by the same neurologist on each occasion. The protocol used was approved by the ethics committee at Huashan Hospital, Fudan University, Shanghai, and all

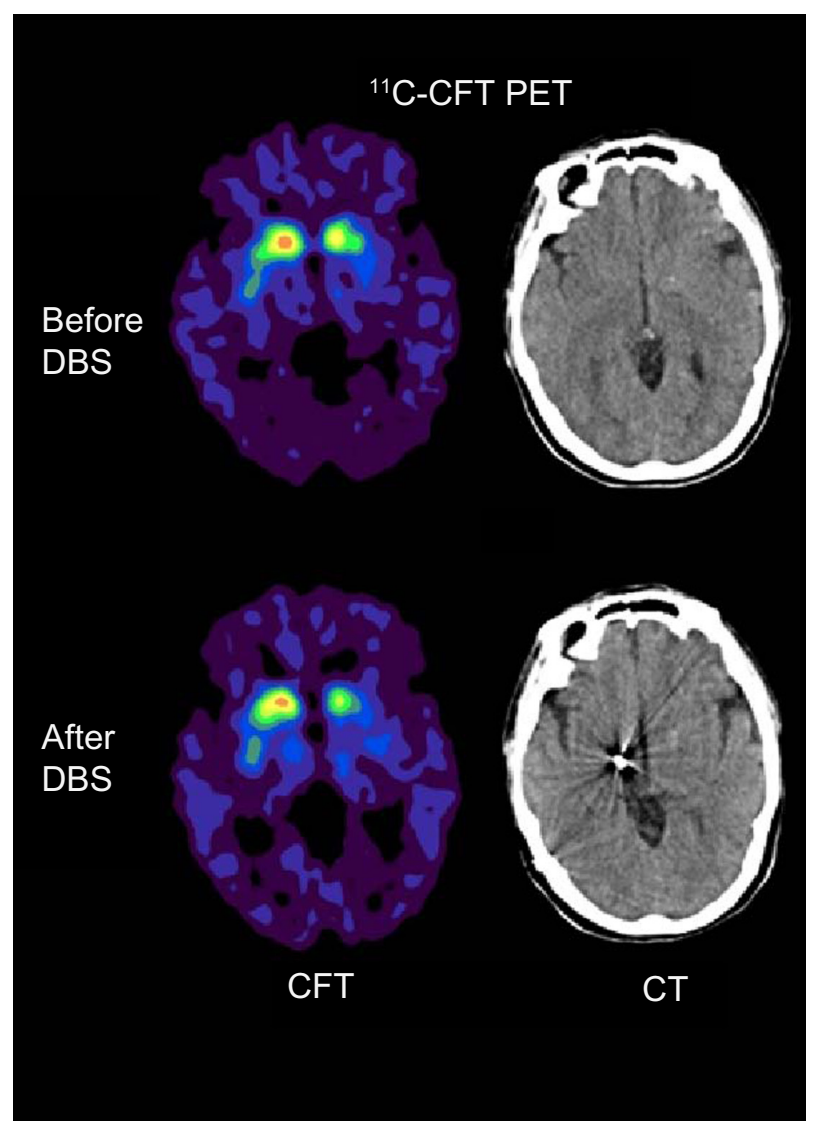

Figure I Comparison of "C-CFT PET imaging before and after deep brain stimulation in patient's right subthalamic nucleus.

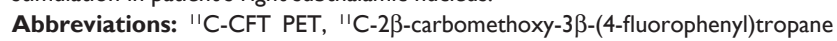
positron emission tomography; CT, computed tomography; DBS, deep brain stimulation. examinations were performed with the patient's informed consent.

\section{Materials and methods}

The patient stopped taking anti-parkinsonian medications at least 12 hours before PET scanning. A $370 \mathrm{MBq}$ injection of ${ }^{11} \mathrm{C}$-CFT was administered intravenously. A 20-minute threedimensional emission scan (Biograph ${ }^{\mathrm{TM}} 64$ HD, Siemens Healthcare, Erlangen, Germany) was acquired 60 minutes post-injection. Attenuation correction was performed using X-ray computed tomography (CT) prior to the emission scan. Following corrections for scatter, dead time, and random coincidences, PET images were reconstructed using threedimensional filtered back projection.

\section{Data analysis}

Anatomically-based volumes of interest for the caudate, anterior putamen, posterior putamen, and occipital cortex were placed manually on the mean image summed over central slices encompassing the striatal structures; these volumes of interest were confirmed for the subject with reference to magnetic resonance images. From the ${ }^{11} \mathrm{C}$-CFT PET scan, we calculated the ratio of specific to nonspecific activity in the caudate, anterior putamen, and posterior putamen volumes of interest by subtracting parieto-occipital activity from striatal activity and dividing by parieto-occipital activity. ${ }^{4}$

\section{Results}

Clinically, DBS had a beneficial effect on the patient's motor symptoms according to her UPDRS and Hoehn and Yahr scores. Her total UPDRS score decreased from 46 to 24 and her Hoehn and Yahr score decreased from 4 to 2. UPDRS decreased from 11 to 8 on the right and from 19 to 6 on the left, indicating more improvement in motor symptoms in the left limbs than in the right limbs.

At baseline, ${ }^{11} \mathrm{C}$-CFT PET showed asymmetrically reduced dopamine transporter binding in the caudate nucleus, and in the anterior and posterior putamen bilaterally (Figure 1). One year after DBS, ${ }^{11} \mathrm{C}$-CFT PET showed further decreases in dopamine transporter binding in the caudate nucleus, and in the anterior and posterior putamen bilaterally. On the stimulated side, the dopamine transporter slope rate was $-0.12,-0.07$ and -0.16 for the anterior putamen, posterior putamen, and caudate, respectively; the annual decrease in dopamine transporter at these sites was $12 \%, 7 \%$, and $16 \%$, respectively, while on the unstimulated side, the respective dopamine transporter slope rate was $-0.16,-0.08$, and -0.13 , respectively, with an annual decrease in dopamine transporter of $16 \%, 8 \%$, and $13 \%$ (Figure 2). The slope rate 


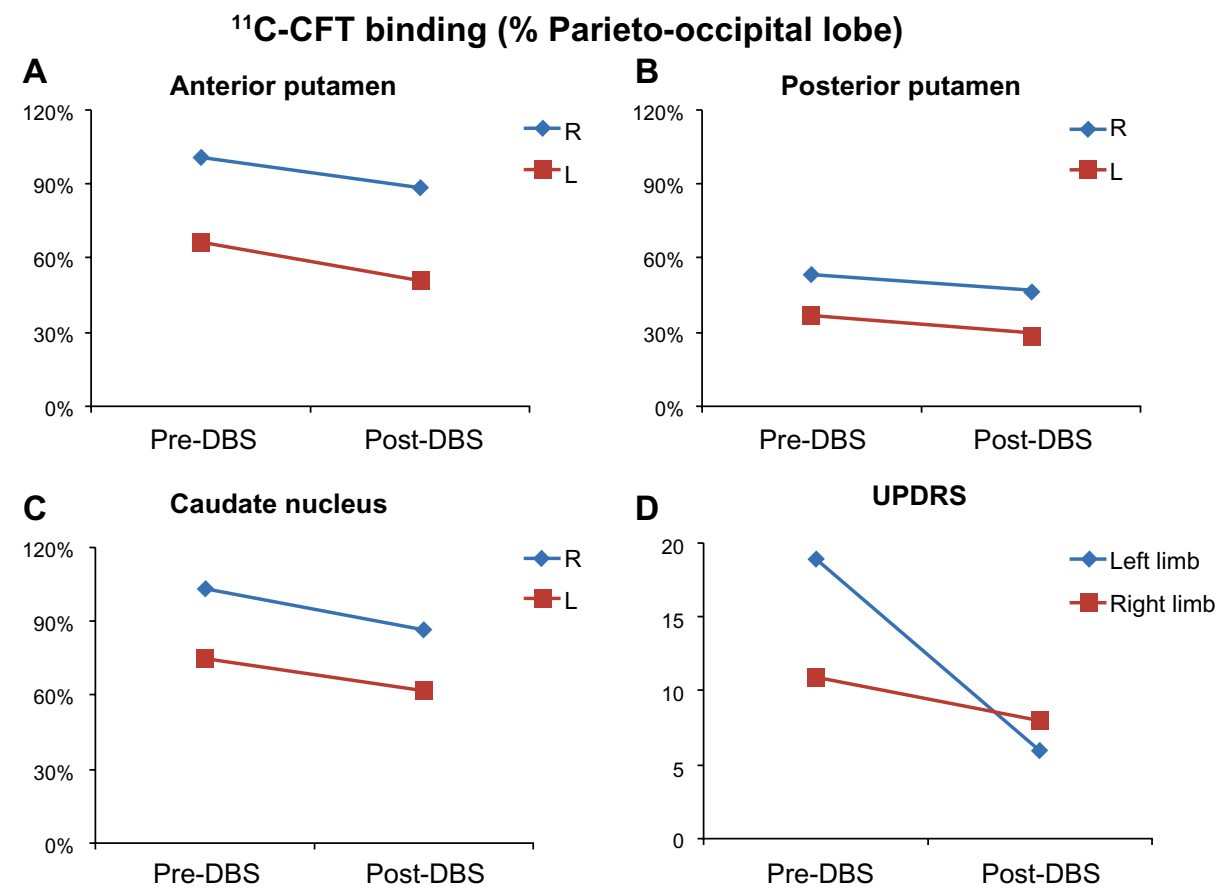

Figure 2 (A-C) Pre-DBS and post-DBS plots demonstrating progressive bilateral decreases (percentage relative to parieto-occipital reference) of post-DBS "C-CFT dopamine transporter binding in the anterior putamen $(\mathbf{A})$, posterior putamen $(\mathbf{B})$ and caudate $(\mathbf{C})$ after one year. Stimulation was performed on the right side. (D) The UPDRS score for the left limbs was similar to that of the right limbs before and after DBS.

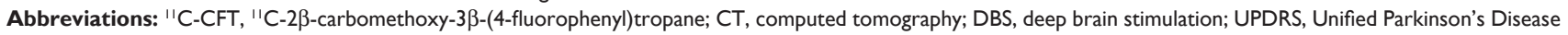
Rating Scale; ${ }^{18} \mathrm{~F}-\mathrm{FDG}$ PET, fluorodeoxyglucose positron emission tomography; STN, subthalamic nucleus; L, left; R, right.

was quite close between the stimulated and unstimulated sides in the three regions, suggesting that degeneration of the dopamine transporter was similar on both sides during the course of one year.

\section{Discussion}

As reported previously, an 11.2\% natural decline of dopaminergic neurons in advanced PD may be expected. ${ }^{5}$ Our patient's annual decline of dopamine transporter distribution on the stimulated side was $7 \%-16 \%$, and was almost equal to the $8 \%-16 \%$ decline found on the unstimulated side, suggesting that DBS to the subthalamic nucleus may not change dopamine transporter distribution. Hesse et al used ${ }^{123}$ I-FP-CIT SPECT to measure dopamine transporter availability and demonstrated that there was no change in striatal ${ }^{123}$ I-FP-CIT binding between pre-DBS and follow-up at one year after DBS. ${ }^{3}$ ${ }^{123}$ I-FP-CIT binding was also found to be reduced to an equal extent in patients who have undergone stimulation of the subthalamic nucleus and nonoperated PD patients with advanced disease. ${ }^{2}$ In ${ }^{18} \mathrm{~F}$-fluorodopa PET studies of presynaptic tracer binding, one demonstrated that the decline in presynaptic dopaminergic function continued in advanced PD patients despite clinically effective bilateral subthalamic nucleus stimulation, ${ }^{1}$ but another could not detect any significant difference between stimulation on and off states. ${ }^{6}$ Consequently, these studies concluded that DBS leading to clinical benefit was not associated with dopamine transporter recovery.

In our case, motor symptom improvement in the right side was not as good as on the left, indicating that DBS to the right subthalamic nucleus have an effect on motor symptoms which was not consistent with the change in dopamine transporter. Therefore, we conclude that clinical benefit after unilateral deep brain stimulation may not be directly associated with dopamine transporter function. Further studies with larger sample sizes are needed to confirm our hypothesis.

\section{Acknowledgment}

This research was supported by the National Natural Science Foundation of China (grant 81171189).

\section{Disclosure}

The authors report no conflicts of interest in this work.

\section{References}

1. Hilker R, Portman AT, Voges J, et al. Disease progression continues in patients with advanced Parkinson's disease and effective subthalamic nucleus stimulation. J Neurol Neurosurg Psychiatry. 2005;76(9): 1217-1221.

2. Lokkegaard A, Werdelin LM, Regeur L, et al. Dopamine transporter imaging and the effects of deep brain stimulation in patients with Parkinson's disease. Eur J Nucl Med Mol Imaging. 2007;34(4):508-516. 
3. Hesse S, Strecker K, Winkler D, et al. Effects of subthalamic nucleus stimulation on striatal dopaminergic transmission in patients with Parkinson's disease within one-year follow-up. J Neurol. 2008;255(7):1059-1066.

4. Tang CC, Poston KL, Dhawan V, Eidelberg D. Abnormalities in metabolic network activity precede the onset of motor symptoms in Parkinson's disease. J Neurosci. 2010;30(3):1049-1056.
5. Marek K, Innis R, van Dyck C, et al. $\left[{ }^{123} \mathrm{I}\right]$ beta-CIT SPECT imaging assessment of the rate of Parkinson's disease progression. Neurology. 2001;57(11):2089-2094.

6. Arai N, Yokochi F, Ohnishi T, et al. Mechanisms of unilateral STNDBS in patients with Parkinson's disease: a PET study. J Neurol. 2008;255(8):1236-1243. 


\section{Supplementary materials Method}

${ }^{18}$ Fluorodeoxyglucose positron emission tomography $\left({ }^{18}\right.$ F-FDG PET) imaging.

\section{Procedure}

${ }^{18} \mathrm{~F}$-FDG PET scans were performed 3 hours after a ${ }^{11} \mathrm{C}-2 \beta$ carbomethoxy-3 $\beta$-(4-fluorophenyl)tropane $\left({ }^{11} \mathrm{C}-\mathrm{CFT}\right) \mathrm{PET}$ scan. The patients were fasted overnight and in the "medication off" and "DBS on" state. A $185 \mathrm{MBq}$ injection of ${ }^{18} \mathrm{~F}-\mathrm{FDG}$ was administered intravenously under standardized conditions (in a quiet, dimly lit room with the patient's eyes open and head immobilized using a head-holder). A 10-minute three-dimensional emission scan was acquired 45 minutes post-injection. The ${ }^{18} \mathrm{~F}-\mathrm{FDG}$ PET scan used the same three-dimensional filtered back projection reconstruction and the same PET scanner as used for ${ }^{11} \mathrm{C}-\mathrm{CFT}$ PET.

\section{Data analysis}

Twelve age-matched healthy female controls were carefully chosen for comparison of their scans with the baseline and second scans of the case patient. The PET images were analyzed using SPM2 software (Wellcome Department of Cognitive Neurology, London, UK) running on Matlab version 2006a (Mathworks Inc., Sherborn, MA, USA). These comparisons were based on a two-tailed unpaired $t$-test and restricted a priori to a volume of interest which included the putamen, caudate nucleus, thalamus, and the cerebellum. This masking drastically reduced the number of voxel-byvoxel statistical comparisons, and a threshold of $P<0.001$ (cluster-corrected at $P<0.05$ ) was considered to be statistically significant. ${ }^{1}$

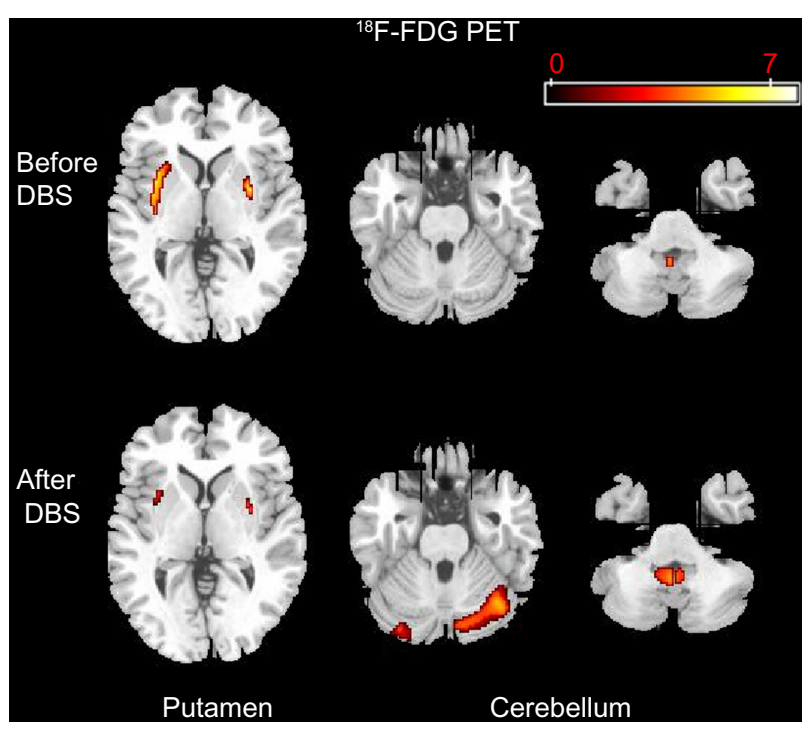

Figure SI Comparison of ${ }^{18} \mathrm{~F}$-FDG PET imaging before and after deep brain stimulation in patient's right subthalamic nucleus.

Abbreviations: ${ }^{18} \mathrm{~F}-\mathrm{FDG} \mathrm{PET}$, fluorodeoxyglucose positron emission tomography; DBS, deep brain stimulation.

\section{Results}

At baseline, ${ }^{18} \mathrm{~F}-\mathrm{FDG}$ PET revealed slightly asymmetrical hypermetabolism bilaterally in the putamen and cerebellar vermis (Figure S1), while at follow-up one year after DBS the extent of FDG hypermetabolism in the putamen decreased bilaterally and the extent of FDG hypermetabolism increased in the cerebellum and cerebellar vermis.

\section{Reference}

1. Wang J, MaY, Huang Z, et al. Modulation of metabolic brain function by bilateral subthalamic nucleus stimulation in the treatment of Parkinson's disease. J Neurol. 2010;257(1):72-78.

\section{Publish your work in this journal}

Neuropsychiatric Disease and Treatment is an international, peerreviewed journal of clinical therapeutics and pharmacology focusing on concise rapid reporting of clinical or pre-clinical studies on a range of neuropsychiatric and neurological disorders. This journal is indexed on PubMed Central, the 'PsycINFO' database and CAS.

\section{Dovepress}

The manuscript management system is completely online and includes a very quick and fair peer-review system, which is all easy to use. Visit http://www.dovepress.com/testimonials.php to read real quotes from published authors. 